\title{
Ameliorative Effect of Captopril and Valsartan on an Animal Model of Diabetic Cardiomyopathy
}

\author{
Chun-Hong Zhang, ${ }^{a, b}$ Jun Lu, ${ }^{a}$ Xiao-Jiang Yu, ${ }^{a}$ Lei Sun, ${ }^{a}$ and Wei-Jin ZAnG ${ }^{*, a, c}$ \\ ${ }^{a}$ Department of Pharmacology, School of Medicine, Xi'an Jiaotong University; ${ }^{b}$ Department of Endocrine, The Second \\ Affiliated Hospital, School of Medicine, Xi'an Jiaotong University; and ${ }^{c}$ Key Laboratory of Environment and Genes \\ Related to Diseases of Ministry of Education, School of Medicine, Xi'an Jiaotong University; Xi'an 710061, P. R. China. \\ Received May 19, 2008; accepted August 4, 2008; published online August 22, 2008
}

\begin{abstract}
The objective of this study was to clarify the relationship between angiotensin II and the pathogenesis of diabetic cardiomyopathy by observing the effects of related drugs on diabetic cardiomyopathy in rats. Captopril and Valsartan, an automatic biochemical analyzer, and radioimmunoassay technology were used to an experimental rat model of diabetic cardiomyopathy to dynamically measure the levels of creatine kinase-MB, lactate dehydrogenase-1 in the serum, and angiotensin I and II in the plasma, and to observe changes in the myocardial ultrastructure. The content of angiotensin I and II was increased and the renin-angiotensin system was in a hyperfunctional state in experimental rats with myocardial damage. Angiotensin-converting enzyme inhibitors and angiotensin II receptor 1 antagonists improved the myocardial structure and cardiac function. It is concluded that hyperfunction of the renin-angiotensin system is involved in the pathogenesis of diabetic cardiomyopathy, with an increase in angiotensin being a key factor. Preventing the increase in angiotensin II or the action of angiotensin II on its receptor can prevent the occurrence and development of diabetic cardiomyopathy.
\end{abstract}

Key words renin-angiotensin system; angiotensin; cardiomyopathy; diabetes; myocardium

Since 1972, when Rubler first put forward the concept of diabetic cardiomyopathy, autopsies ${ }^{1,2)}$ and clinical manifestations $^{3-6)}$ have shown that diabetics do indeed exhibit a pathological state of specific diabetic cardiomyopathy. The characteristic myocardial pathological manifestations of diabetic cardiomyopathy are cardiac hypertrophy and interstitial fibrosis, ${ }^{7,8)}$ which result in restrictive cardiomyopathy being characterized mainly by impaired systolic function.

The pathogenesis of diabetic cardiomyopathy has not been confirmed, but it is mainly attributed to the metabolism and $\mathrm{Ca}^{2+}$ transport and regulation. Some scholars have suggested that the glucose metabolism disorder in myocardial tissue associated with chronic diabetes mellitus (DM) is due to impaired glucose transport and enhanced fatty acid metabo$\mathrm{lism}^{9,10)}$ resulting from insulin deficiency. Previous studies found that the exchange of $\mathrm{Ca}^{2+}$, the $\mathrm{Na}^{+}-\mathrm{Ca}^{2+}$ exchange, and the enzymatic activities of $\mathrm{Na}^{+}, \mathrm{K}^{+}$, and ATP are clearly decreased in the myocardial cell membranes of DM rats and the activity of $\mathrm{Ca}^{2+}$ in sarcoplasmic reticulum is decreased, leading to intracellular $\mathrm{Ca}^{2+}$ overload and disturbance to the respiration function of mitochondria, thus causing a decline in cardiac function. ${ }^{11-14)}$

The discoveries of renin by Tigerstedt et al. in 1898 and angiotensin by Braun-Menendez et al. in 1939 opened up research into the renin-angiotensin system (RAS). The RAS and the cardiovascular system are closely related, with the former playing an important role in cardiac hypertrophy and interstitial fibrosis. Angiotensin II is an important bioactive substance of the RAS, exerting major effects on the blood volume, hemodynamics, and the regulation of homeostasis. ${ }^{15)}$ Under physiological conditions, angiotensin II has a positive inotropic effect on the myocardium, promoting sympathetic nerve excitation, maintaining angiostasis, and regulating coronary flow and the heart rate. Under pathological conditions, angiotensin at a concentration above normal physiological levels may cause the release of catecholamines through the sympathetic nerve system or have a direct effect on the heart, thus resulting in cardiac hypertrophy. Angiotensin II acts mainly via its receptor. Previous studies have indicated $^{16-18)}$ that there are many high-affinity angiotensin II binding sites on the surface of human and rat cardiac fibroblasts, most of which are the 1 type angiotensin II receptor $\left(\mathrm{AT}_{1} \mathrm{R}\right)$. The promotion of cardiac hypertrophy by angiotensin II is mediated mainly by cardiac fibroblasts. Angiotensin II can also induce the expression of proto-oncogene, ${ }^{19)}$ activate the expression of some peptide growth factors, and participate in the occurrence of cardiac hypertrophy. Furthermore, angiotensin II can promote the occurrence of cardiac interstitial fibrosis by regulating fibrotic growth factors. $^{20-24)}$

Given that the characteristic myocardial pathological manifestations of diabetic cardiomyopathy are cardiac hypertrophy and interstitial fibrosis, does the RAS - which is closely related to cardiac hypertrophy and interstitial fibrosis-also play a role in the pathogenesis of diabetic cardiomyopathy, and, if so, what is the underlying mechanism? This prompted us to establish an animal model of diabetic cardiomyopathy. We used an angiotensin-converting enzyme inhibitor (ACEI) and a 1 type angiotensin II receptor blocker $\left(\mathrm{AT}_{1} \mathrm{RB}\right)$ to determine whether the main bioactive substance in the RAS, angiotensin II, is related to the occurrence and development of diabetic cardiomyopathy, with the aim of elucidating the relationship between angiotensin and diabetic cardiomyopathy and providing a theoretical basis for the prevention and control of diabetic cardiomyopathy.

\section{MATERIALS AND METHODS}

Experimental Animals A total of 120 healthy SpragueDawley rats (SD) from the Animal Experimental Center of Medical College at Xi'an Jiaotong University were housed individually in cages. Rats were used in accordance with the Guidelines on the Care and Use of Laboratory Animals issued by the Chinese Council on Animal Research and the 
Guidelines of the Animal Care. The study was approved by the ethical committee of Xi'an Jiaotong University. The females (60) and males (60) weighed 245.13 \pm 16.53 and $273.65 \pm 15.32 \mathrm{~g}$, respectively. They received a normal diet and tap water.

Preparation of the Rat Model with Diabetic Cardiomyopathy Rats were fed for 13 weeks. In week 1 they received a normal diet for adaptation to the environment and stabilization of their mood. In week 2 they received streptozotocin (STZ). In week 3 we measured the experimental indices and performed the grouping. The animals were medicated from the fifth week ( 3 weeks after the injection of STZ). The rats were divided randomly into the following groups:

1. Normal group: Normal SD rats subject to no experimental intervention.

2. Control group: Normal SD rats injected once with a constant volume of buffer solution $(0.1 \mathrm{~mol} / 1$ citrate buffer solution, $\mathrm{pH} 4.2$ ).

3. Diabetic cardiomyopathy group: Rats with DM that received one dosage via the intraperitoneal injection of $55 \mathrm{mg} / \mathrm{kg} \mathrm{STZ}$ in fresh buffer solution.

4. Captopril group: Rats with DM that received Captopril by intragastric administration from week 5 .

5. Valsartan group: Rat with DM that received Valsartan by intragastric administration from week 5.

6. Combination insulin and Captopril group: Rats with DM that received Captopril by intragastric administration from week 5 , and were simultaneously subcutaneous injected with long-acting protamine zinc insulin.

7. Combination insulin and Valsartan group: Rats with DM that received Valsartan by intragastric administration from week 5, and were simultaneously subcutaneously injected with long-acting protamine zinc insulin.

Construction of the Model: After fasting for $24 \mathrm{~h}$, newly prepared STZ solution was injected into rats via the left inferior celiac artery. After stabilizing for 1 week, rats with a fasting glucose of $>11.1 \mathrm{mmol} / 1$ and a nonfasting glucose of $>16.7 \mathrm{mmol} / \mathrm{l}$ were assigned to the DM group. Three weeks after being injected STZ, diabetic rats were medicated when the pathology of diabetic cardiomyopathy occurred. ${ }^{25)}$

Administration: The glucose condition of the rats determined the hypodermic dosage of insulin, and the average daily dosage for each was 2-4 U. Meanwhile, the Captopril dosage was based on a surface area conversion formula, with the average dosage being $13 \mathrm{mg} / \mathrm{kg}$. The Valsartan dosage was $14 \mathrm{mg} / \mathrm{kg}$

Detection of Glucose Condition: Glucose was detected using Johnson \& Johnson's One Touch Profile Blood Glucose Meter. The myocardial tissue was extracted from some animals at weeks $3,4,5,9$, and 13 and examined by electron microscopy. Creatine kinase-MB (CK-MB) and lactate dehydrogenase-1 (LDH-1) levels in rat serum were detected using an automatic biochemical analyzer. Plasma Angiotensin I and II were measured by use of high-performance liquid chromatography combined with a sensitive radioimmunoassay. ${ }^{26)}$

Electron Microscopy Specimen Cutting and Preparation After anesthetizing with 3\% sodium pentobarbital, the chest of the rat was opened to reveal the heart, which was rapidly removed. At a low temperature, a specimen of the left ventricular myocardium removed with ophthalmic scissors was cut into a $1 \mathrm{~mm}$ tissue mass. Images were taken after fixation, soaking, stepwise alcohol dehydration, displacement, embedding, polymerization, sectioning, and staining.

Statistical Analysis One-way factorial ANOVA was used for statistical analysis. The results were expressed as means \pm S.E.M., and $p<0.05$ was used as the criterion for statistical significance.

\section{RESULTS}

Preparation of Animal Model with Diabetic Cardiomyopathy After being injected with STZ, rats in the DM group presented with dull fur, hair loss, becoming dirty easily, inactivity, polydipsia, polyuria, and polyphagia, but loss of weight. Before establishing the model, the average fasting blood glucose of the rats in each group was $2-3 \mathrm{mmol} / \mathrm{l}$. At $4 \mathrm{~d}$ after the injection of STZ, the fasting blood glucose in both the normal and control groups was normal. The fasting and nonfasting blood glucose levels of the rats in the experimental group were $13.5-22.8$ and $16.0-35.1 \mathrm{mmol} / \mathrm{l}$, respectively, with this hyperglycemia existing continuously and stably. After being injected for 3 weeks, the observed myocardial structure of some rats in the experiment group was consistent with the pathological presentation of diabetic cardiomyopathy. ${ }^{25)}$

Serum Levels of Cardiac-Specific Enzymes In this experiment, the normal scope of CK-MB was less than $25 \mathrm{U} / 1$, and the normal range of LDH-1 was from 114 to $240 \mathrm{U} / 1$.
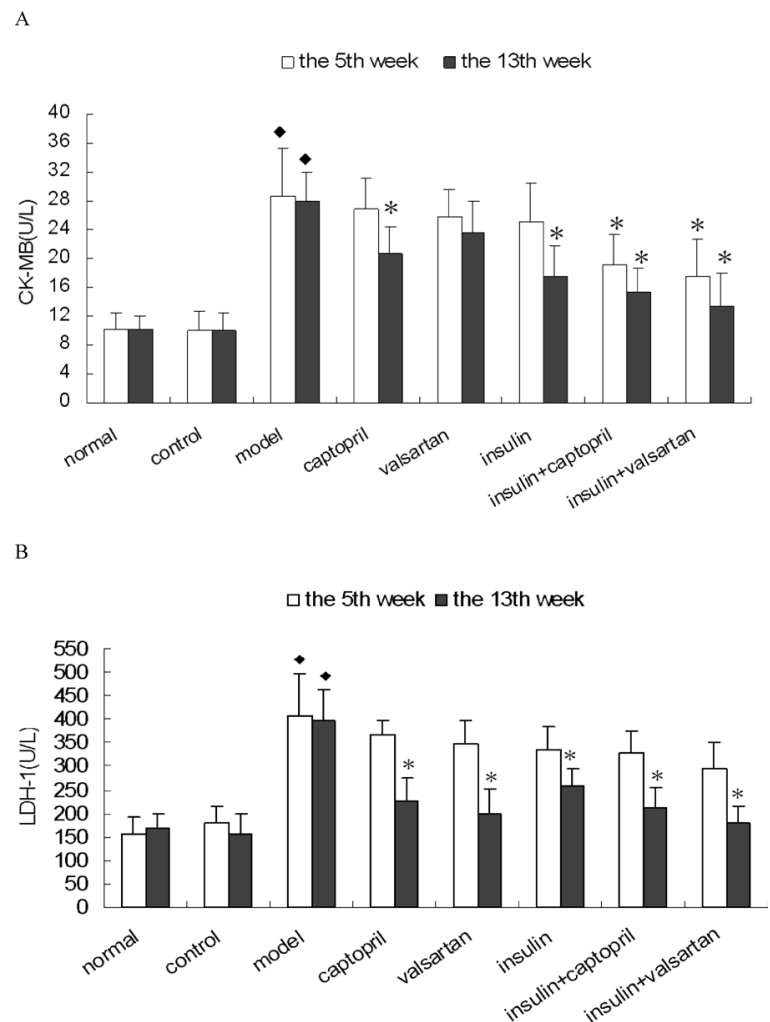

Fig. 1. Levels of CK-MB and LDH-1 in Rats at Weeks 5 and 13

Horizontal axis indicates the rat group, and vertical axis indicates the CK-MB and LDH-1 levels. Comparisons of CK-MB and LDH-1 levels at weeks 5 and 13 between the model and control groups $(\bullet p<0.05)$ and between the treatment and model groups $(* p<0.05)$. Panels A and B show the mean CK-MB and LDH-1 levels in serum at weeks 5 and 13 , respectively. 
The detection results are shown in Fig. 1, which indicates that (1) CK-MB and LDH-1 were significantly higher in the model group than in the control group $(p<0.05)$, (2) the treatments lowered $\mathrm{CK}-\mathrm{MB}$ and $\mathrm{LDH}-1$ in each treated group versus the model group $(p<0.05)$, and there is no difference among the treated groups.

Angiotensin I and II The detection results of angiotensin I shown in Fig. 2 indicate that (1) from week 5 of the trial (i.e., 3 weeks after the injection of STZ), the angiotensin I level was significantly higher in the model group than in the control group, with this lasting until week 13, and (2) the treatments had no obvious effects on angiotensin I levels.

The detection results of angiotensin II show that (1) from week 5 of the trial, the angiotensin II level was clearly higher in the model group than in the control group $(p<0.05)$, with this lasting until week 13, (2) the angiotensin II level was significantly lower in the Captopril group and insulin group than in the model group $(p<0.05)$, with Valsartan treatment only and combination treatment of insulin and Valsartan having no significant influence on the angiotensin II level, and (3) the angiotensin II level decreased more in the combination treatment of insulin and Captopril than in the model group $(p<0.05)$.

Myocardial Ultrastructure The myocardial structure of the rats in the normal and control groups was clearly evident. Myofibrils are the main components of cytoplasm, and they appeared orderly, with bright and dark areas clearly evident.

A

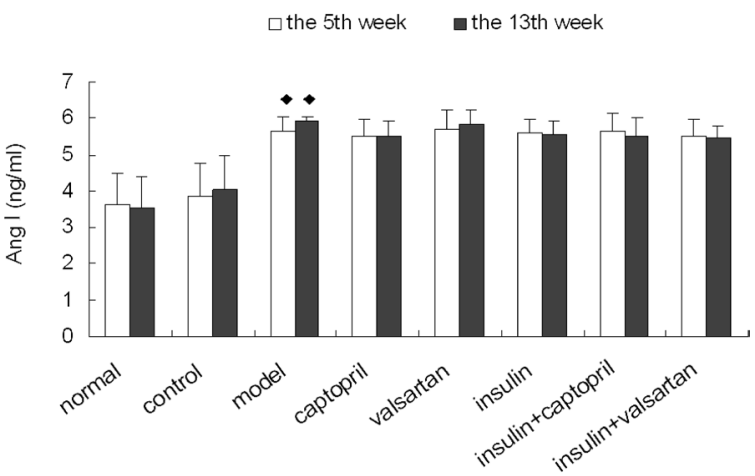

B

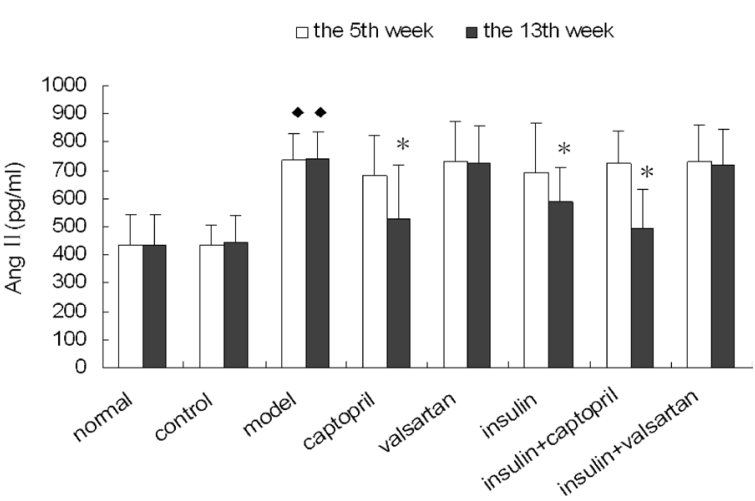

Fig. 2. Average Angiotensin I and II Levels in Each Rat Group

Horizontal axis indicates the rat group, and vertical axis indicates the angiotensin I and II levels. Comparisons of angiotensin I and II levels at weeks 5 and 13 between the model and control groups $(\bullet p<0.05)$ and between the prevention/treatment and model groups $(* p<0.05)$. Panels A and B show the mean angiotensin I levels in serum at weeks 5 and 13 , respectively.
The myocardial structure in the model group was characterized by obvious reductions in myofibril content, breaking, twisting, and disorderly myofilaments. Focal and compensatory states of mitochondria increased, and in different courses of diseases presented as swelling, deformation, shortening, cristal breaking, and even vacuolization. The sarcoplasmic reticulum expanded and mass vacuoles formed. Progression of DM resulted in worsening cardiomyopathy, which may lead to the focal necrosis of myocardial fibers and the dissolution or even disappearance of myofilaments. From week 5 there was clear cardiomyopathy present. Medication resulted in the improvement in the myocardial structure being greater in each experiment group than in the model group (Fig. 3).

\section{DISCUSSION}

There are so many diabetic patients worldwide. The biggest hazard of DM to humans is the occurrence of serious complications involving multiple systems and viscera of the entire body. Therefore, delaying or preventing the development of these complications is becoming increasingly important.

It has been shown that the pathological presentations of diabetic cardiomyopathy are cardiac hypertrophy and intersti-

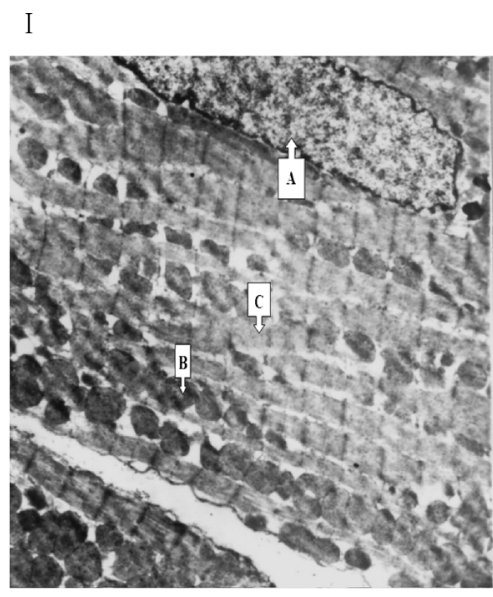

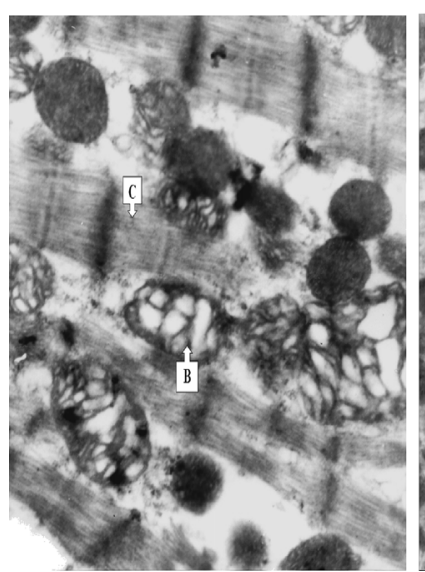

III

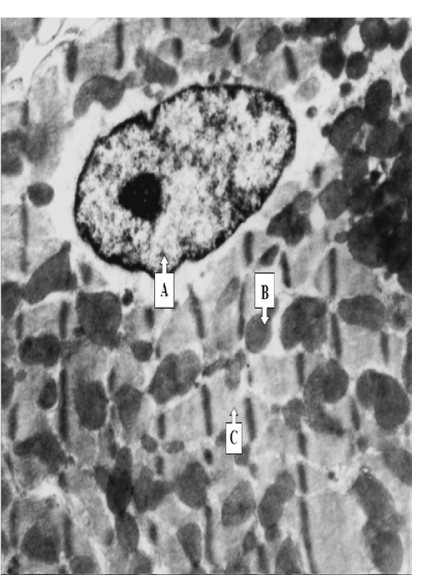

Fig. 3. Image of the Myocardial Ultrastructure in the Control, Model, and Insulin + Valsartan Groups

$\mathrm{A}$ indicates cell nucleus, $\mathrm{B}$ indicates mitochondria, and $\mathrm{C}$ indicates myofibril (myofilaments). Myocardial structure in the control group (I, $\times 4000$; II, $\times 15000)$ and in the insulin + Valsartan group (III, $\times 4000)$. 
tial fibrosis. ${ }^{27)}$ The close relationships of cardiac hypertrophy and interstitial fibrosis with the RAS have been confirmed. Angiotensin II is an important bioactive substance of the RAS and plays an important role in the pathogenesis of interstitial fibrosis and cardiac hypertrophy. ${ }^{28,29)}$ We investigated whether angiotensin II is a key factor in the close relationship between the pathological manifestation of diabetic cardiomyopathy and the RAS, and showed that angiotensin II affects the pathogenesis of diabetic cardiomyopathy. We also revealed the myocardial protection effects on rats with diabetic cardiomyopathy by applying $\mathrm{ACEI}$ and $\mathrm{AT}_{1} \mathrm{RB}$, thus offering evidence of putative clinically effective medications.

When Diabetic Cardiomyopathy Occurs, the RAS Is in a Hyperfunctional State We chemically induced diabetic cardiomyopathy in SD rats by applying STZ, and found that the angiotensin I and II levels were both higher in the model group than in the control group, indicating that the RAS is in a hyperfunctional state during diabetic cardiomyopathy.

The RAS has short-term functions on the circulatory and endocrine system to regulate blood pressure and water and electrolyte balance. It also controls the tissue distribution of endocrine, paracrine, and intracellular autocrine throughout the body, which mainly exerts long-term effects such as hypertension progression and blood vessel and cardiac hypertrophy. The heart is one of the target organs of the RAS, but there is spatial variation in heart activity, with this being highest in the right atrium, followed by the right ventricle, left atrium, and left ventricle.

The target tissue of this research was the myocardium of the rat left ventricle, where the RAS has the least activity, which tends to exhibit damage of RAS hyperfunction to the myocardium of diabetic cardiomyopathy. The research shows that the deterioration of a rat with diabetic cardiomyopathy results in an increase in the angiotensin I and II levels, which is indicative of RAS hyperfunction.

Angiotensin II Is a Key Factor in the Pathogenesis of Diabetic Cardiomyopathy It is clarified that the roles played by the RAS in maintaining water and electrolyte balances and regulating blood pressure. It is known that angiotensin II is the main bioactive component in the RAS. ${ }^{30}$ Captopril and Valsartan were chosen as long-term medications aimed at preventing the formation of angiotensin II, and thereby reducing the effect of angiotensin II on target issue. Observing whether the cardiac structure of the rat with diabetic cardiomyopathy can be improved and whether angiotensin II is a key factor in the pathogenesis of diabetic cardiomyopathy would help to clarify the pathogenesis.

This research showed that: damage to the myocardial ultrastructure of rats with diabetic cardiomyopathy can be reduced by treatment with Captopril or Valsartan. Relieving RAS hyperfunction, especially reducing the formation of angiotensin II, or stopping the effects of angiotensin II by preventing it from combining with its receptor, can block the development of myocardial pathology in rats with diabetic cardiomyopathy. This indicates that angiotensin II is also a key factor in the pathogenesis of diabetic cardiomyopathy.

Basis for Choosing an ACEI Blocking any link in the RAS chain that generates angiotensin II can inhibit the effects of the RAS. The ACEI we chose was effective at preventing the generation of angiotensin II. There are several mechanisms by which ACEIs can protect the cardiovascular system. $^{31)}$ First, they can protect the myocardium. Second, they have the effect of anti-myocardial ischemia. Third, they can protect blood vessels. Therefore, ACEIs have been widely used in the treatment and prevention of congestive heart failure (CHF). In its early stage, ACEIs can prevent the development of ventricle dysfunction and reconstruction, while in the later stage they can reverse heart proliferation, reconstruction, and dysfunction and thereby enhance the survival rate.

In our experiments Captopril prevented the generation of angiotensin II and the serum content of angiotensin II decreased, and the pathological lesion of diabetic cardiomyopathy was reduced, which shows that an increased concentration of angiotensin II in the RAS is a key factor in the pathogenesis of diabetic cardiomyopathy. Therefore, reducing the generation of angiotensin II by applying ACEI drugs can effectively prevent the development of the pathogenesis of diabetic cardiomyopathy.

Choice of $\mathbf{A T}_{\mathbf{1}} \mathbf{R B}$ Drug We chose an $\mathrm{AT}_{1} \mathrm{RB}$ drug (Valsartan) due to the incomplete effect that ACEIs have on blocking angiotensin II; in contrast, an $\mathrm{AT}_{1} \mathrm{RB}$ can specifically prevent angiotensin II from exerting its function. Moreover, patients often give up using ACEIs due to the side effect of dry cough. An $\mathrm{AT}_{1} \mathrm{RB}$ is therefore more efficient, safer, and has better compliance than an ACEI.

$\mathrm{AT}_{1} \mathrm{RB}$ drugs exert similar clinical pharmacological effects. ${ }^{32-34)}$ At present, clinically used $\mathrm{AT}_{1} \mathrm{RBs}$ belong to nonpeptide drugs, which have the same specificity in preventing angiotensin II from combining its receptor. However, in terms of their strengths and selectivity, the $\mathrm{AT}_{1} \mathrm{R}$ and $\mathrm{AT}_{2} \mathrm{R}$ differ in ratio and bioactive substances. According to the detection conditions, the selective block by sartan drugs is 1000 times stronger for $\mathrm{AT}_{1} \mathrm{R}$ than for $\mathrm{AT}_{2} \mathrm{R}$, among which Valsartan has the best strength ratio. ${ }^{31)}$ Therefore, we chose to use Valsartan.

This research suggests that Valsartan-based drugs can effectively prevent angiotensin II from acting on its receptor and radically relieve the adverse effects that angiotensin II has on the myocardium at pathological concentrations, the occurrence of cardiac interstitial fibrosis and cardiac hypertrophy of the rats with diabetic cardiomyopathy can be effectively prevented. The above has further proved that angiotensin II can induce and promote the development of diabetic cardiomyopathy at pathological concentrations.

\section{CONCLUSION}

The experiments of this study have shown the effects that angiotensin II have on the pathogenesis of diabetic cardiomyopathy: there is a close relationship between RAS hyperfunction and the pathogenesis of diabetic cardiomyopathy, the increased content of angiotensin II plays a vital role in the development of diabetic cardiomyopathy, and an $\mathrm{AT}_{1} \mathrm{RB}$ can effectively prevent angiotensin II from combining with its receptor to control the development of diabetic cardiomyopathy. However, whether applying $\mathrm{AT}_{1} \mathrm{RB}$ drugs in the early stage of DM can prevent the occurrence of diabetic cardiomyopathy still needs clinical clarification.

Acknowledgements This work was supported by grants from the National Basic Research Program of China (973 
Program) (No. 2007CB512005), the National Natural Science Foundation of China (No. 30770785), the Cultivation Fund of the Key Scientific and Technical Innovation Project of Chinese Ministry of Education (No. 705045) and the Specialized Research Fund for the Doctoral Program of Higher Education (No. 20050698012).

\section{REFERENCES}

1) Regan T. J., Lyons M. M., J. Clin. Invest., 60, 885-899 (1977).

2) David S. H., Diabetes Care, 18, 708 -714 (1995).

3) Pérez J. E., McGill J. B., Santiago J. V., Schechtman K. B., Waggoner A. D., Miller J. G., Sobel B. E., J. Am. Coll. Cardiol., 19, 1154-1162 (1992).

4) Hardin N. J., Coron. Artery. Dis., 7, 99-108 (1996).

5) Lind L., Berne C., Andrén B., Lithell H., Diabetologia, 39, 16031606 (1996).

6) Di Bello V., Giampietro O., Matteucci E., Giorgi D., Bertini A., Piazza F., Talini E., Paterni M., Giusti C., G. Ital. Cardiol., 28, 1128-1137 (1998).

7) Rodrigues B., Cam M. C., McNeill J. H., Mol. Cell. Biochem., 186, 53-57 (1998).

8) Tziakas D. N., Chalikias G. K., Kaski J. C., Coron. Artery Dis., 16 (Suppl. 1), S3-S10 (2005).

9) Meyer C., Schwaiger M., Am. J. Cardiol., 80, 94A-101A (1997).

10) Carley A. N., Severson D. L., Biochim. Biophys. Acta, 1734, 112-126 (2005).

11) Schaffer S. W., Ballard-Croft C., Boerth S., Allo S. N., Cardiovasc. Res., 34, 129-136 (1997).

12) Yu J. Z., Rodrigues B., McNeill J. H., Cardiovasc. Res., 34, 91-98 (1997).

13) Cesario D. A., Brar R., Shivkumar K., Endocrinol. Metab. Clin. North. Am., 35, 601-610, ix-x (2006).

14) Zhao X. Y., Hu S. J., Li J., Mou Y., Chen B. P., Xia Q., J. Physiol. Biochem., 62, 1-8 (2006).

15) Chang R. S., Lotti V. J., Life Sci., 49, 1485-1490 (1991).
16) Itoh H., Mukoyama M., Pratt R. E., Gibbons G. H., Dzau V. J., J. Clin. Invest., 91, 2268-2272 (1993).

17) Sadoshima J.-I., Izumo S., Circ. Res., 73, 413-417 (1993).

18) Kim N. N., Villarreal F. J., Printz M. P., Lee A. A., Dillmann W. H., Am. J. Physiol., 269, E426-E437 (1995).

19) Karin M., J. Biol. Chem., 270, $16483-16486$ (1995).

20) Couse J. F., Bunch D. O., Lindzey J., Schomberg D. W., Korach K. S., Endocrinology, 140, 5855-5865 (1999).

21) Grundy S. M., Benjamin I. J., Burke G. L., Chait A., ECK-MBel R. H., Howard B. V., Mitch W., Smith S. C., Sowers J. R., Circulation, 100, 1134-1146 (1999).

22) Gurantz D., Cowling R. T., Villarreal F. J., Circ. Res., 85, 272-279 (1999).

23) Kato K., Chapman D. C., Rupp H., Lukas A., Dhalla N. S., J. Appl. Physiol., 86, 812-818 (1999).

24) Scheidegger K. J., Dut J., Delafontaine P., J. Biol. Chem., 274, 3522 3530 (1999).

25) Zhang C. H., Zang W. J., Xu J., Yu X. J., Lu J., Jing A. Y., Hu H., Sun Q., Chen L. N., J. Hyg. Res., 35, 703-708 (2006).

26) Huang H., Baussant T., Reade R., Michel J. B., Corvol P., Clin. Exp. Hypertens., 11, 1535-1548 (1989).

27) Goyal R. K., Satia M. C., Bangaru R. A., Gandhi T. P., J. Cardiovasc. Pharmacol., 32, 317-322 (1998).

28) Liu Y. H., Xu J., Yang X. P., Yang F., Shesely E., Carretero O. A., Hypertension, 39, 375-381 (2002).

29) Huentelman M. J., Grobe J. L., Vazquez J., Stewart J. M., Mecca A. P., Katovich M. J., Ferrario C. M., Raizada M. K., Exp. Physiol., 90, $783-790$ (2005)

30) Daniels D., Yee D. K., Fluharty S. J., Exp. Physiol., 92, 523-527 (2007).

31) Tan L. B., Williams S. G., Goldspink D. F., Int. J. Cardiol., 94, 137141 (2004).

32) Gifford R. W. Jr., Med. Clin. North. Am., 81, 1319-1333 (1997).

33) Brede M., Roell W., Ritter O., Wiesmann F., Jahns R., Haase A., Fleischmann B. K., Hein L., Hypertension, 42, 1177-1182 (2003).

34) Talukder M. A., Fujiki T., Morikawa K., J. Cardiovasc. Pharmacol., 44, 557-563 (2004). 